\title{
A MATRIX REPRESENTATION OF GRAPHS AND ITS SPECTRUM AS A GRAPH INVARIANT
}

\author{
David Emms \\ Department of Computer Science \\ University of York, York YO10 5DD, U.K. \\ demms@cs.york.ac.uk \\ Edwin R. Hancock \\ Department of Computer Science \\ University of York, York YO10 5DD, U.K. \\ erh@cs.york.ac.uk \\ Simone Severini \\ Department of Mathematics and Department of Computer Science \\ University of York, York YO10 5DD, U.K. \\ ss540york.ac.uk \\ Richard C. Wilson \\ Department of Computer Science \\ University of York, York YO10 5DD, U.K. \\ richard.wilson@cs.york.ac.uk
}

Submitted: Nov 16, 2005; Accepted: Mar 16, 2006; Published: Apr 4, 2006

2000 Mathematics Subject Classification: 05E30, 05C60

\begin{abstract}
We use the line digraph construction to associate an orthogonal matrix with each graph. From this orthogonal matrix, we derive two further matrices. The spectrum of each of these three matrices is considered as a graph invariant. For the first two cases, we compute the spectrum explicitly and show that it is determined by the spectrum of the adjacency matrix of the original graph. We then show by computation that the isomorphism classes of many known families of strongly regular graphs (up to 64 vertices) are characterized by the spectrum of this matrix. We conjecture that this is always the case for strongly regular graphs and we show that the conjecture is not valid for general graphs. We verify that the smallest regular graphs which are not distinguished with our method are on 14 vertices.
\end{abstract}




\section{Introduction}

Graphs are often conveniently represented using matrices, for example, the adjacency matrix, the Laplacian matrix, etc. [5]. Many important properties of a graph are encoded in the eigenvalues of the matrix representation. However, eigenvalues generally fail to separate isomorphism classes. In this paper, we consider some matrix representations inspired by the notion of coined quantum walks [1]. Since strongly regular graphs give rise to relatively large sets of non-isomorphic graphs which are cospectral with respect to the commonly used matrix representations, we use these graphs as a testing ground for the representations that we define. Let $G$ be a graph and let $U$ be the orthogonal matrix inducing a coined quantum walk on $G$, where the coins are Grover matrices. For every given $k$, we define a digraph, $D$, with an $\operatorname{arc}(i, j)$ if and only if $U_{i, j}^{k}>0$. For $k=1$, we express the adjacency eigenvalues of $D$ in terms of the ones of $G$. When considering strongly regular graphs, we describe how to construct directly $D$ from $G$. For strongly regular graphs, we conjecture that the eigenvalues of the adjacency matrix of $D$ distinguish $G$ from its cospectral mates for $k=3$. We verify this conjecture numerically for all strongly regular graphs up to 64 vertices contained in the tables of Spence [14] (the graphs $\operatorname{srg}(16,9,4,6)$ were obtained from the tables of McKay [9]). By providing counterexamples, we show that our method fails to distinguish general graphs. Hopefully these counterexamples will help understanding what graphs are cospectral with respect to the representations described here.

The remainder of the paper is organized as follows. In Section 2, we recall the definition of the orthogonal matrix $U$ and give a formula for its eigenvalues. We define digraphs derived from powers of $U$, and propose using these digraphs to distinguish the original graph from its cospectral mates. In Section 3, we focus on strongly regular graphs. We describe some structural properties of the digraph $D$ obtained from $U^{3}$. In Section 4 , we list the sets of strongly regular graphs for which the eigenvalues of $D$ successfully distinguish a graph from its cospectral mates. We conclude with counterexamples involving general graphs.

Recently, dynamical processes based on quantum evolution have been considered for attacking the graph isomorphism problem [6,13]. In this context, Shiau et al. [13] gave evidence that quantum walks alone are not successful. The setting they describe is as follows. Let $\{|j\rangle: 1 \leq j \leq n\}$ be an orthonormal basis of a Hilbert space $(|j\rangle$ is a ray and $\langle i|$ is the linear functional which maps each $|j\rangle$ to the usual inner product, which is denoted by $\langle i \mid j\rangle)$. Given a graph $G$, let us define the Hamiltonian $H=-\sum M(G)_{a, b} c_{a}^{\dagger} c_{b}$, where the operator $c_{a}^{\dagger} c_{b}$ is given by $\left\langle i\left|c_{a}^{\dagger} c_{b}\right| j\right\rangle=\delta_{i, a} \delta_{b, j}$ and $M(G)$ is the adjacency matrix of $G$. The evolution for a time $t$ of the initial states $\left\{\left|\psi_{j}(0)\right\rangle=|j\rangle: 1 \leq j \leq n\right\}$ is

governed by the Schrödinger equation $i \frac{d\left|\psi_{j}(0)\right\rangle}{d t}=H\left|\psi_{j}(0)\right\rangle$. In [13], it was shown that the matrix whose entries are $O_{i, j}=\left\langle\psi_{i}(0) \mid \psi_{j}(t)\right\rangle$ do not help in distinguishing pairs of strongly regular graphs with the same set of parameters. However, by making use of some techniques described in [11], it was also given an alternative method which distinguished 
numerically all known strongly regular graphs up to 29 vertices. It would be interesting to investigate potential connections between [13] and the present paper.

\section{Representing graphs with orthogonal matrices}

Let $G=(V, E)$ be a simple undirected graph (that is $G$ is loopless and without multiple edges). Let $D_{G}=(V, A)$ be the directed graph obtained from $G$ by replacing every edge $\{i, j\} \in E(G)$ with the pair of $\operatorname{arcs}(i, j)$ and $(j, i)$. The degree of a vertex $i$ is denoted by $d(i)$. A graph is said to be $k$-regular if every of its vertices has degree $k$. Unless otherwise stated, in this paper we will consider only graphs with minimum degree 3.

Definition 2.1 Given a graph $G$, we denote by $U(G)$ the matrix defined as follows:

$$
\begin{gathered}
\text { for all }(i, j),(k, l) \in A\left(D_{G}\right), \\
U(G)_{(i, j),(k, l)}:= \begin{cases}\frac{2}{d(j)}-\delta_{i, l}, & \text { if } j=k ; \\
0, & \text { otherwise. }\end{cases}
\end{gathered}
$$

The matrix $U(G)$ induces a coined quantum walk on $G$, where the coins are Grover matrices [1]. Here are some basic observations about $U(G)$ :

- If $|E(G)|=m$ then $U(G)$ is $2 m \times 2 m$ and the vertices of degree zero do not contribute to the dimension of $U_{G}$.

- Given $i \in V(G)$, if $d(i)=1$ and $\{i, j\} \in E(G)$, then $U(G)_{(j, i),(i, j)}=1$, and this is the unique non-zero entry in the row indexed by $(j, i)$ and in the column indexed by $(i, j)$.

- Given $j \in V(G)$, if $d(j)=2$ and $\{i, j\},\{j, k\} \in E(G)$, then $U(G)_{(i, j),(j, i)}=$ $U(G)_{(k, j),(j, k)}=0$ and $U(G)_{(i, j),(j, k)}=U(G)_{(k, j),(j, i)}=1$. So, if $G$ is 2-regular, the matrix $U(G)$ has exactly a one in every row and every column and it is then a permutation matrix.

- Since $G$ is undirected, $U(G)_{(i, j),(j, i)} \neq 0$ and $U(G)_{(j, i),(i, j)} \neq 0$ for every $\{i, j\} \in$ $E(G)$. However, we have assumed that the minimum degree of $G$ is 3 , and then $U(G)$ is not symmetric. In fact, for three distinct vertices $i, j, l \in V(G)$ such that $\{i, j\},\{j, l\} \in E(G)$, we have $U(G)_{(i, j),(j, l)} \neq 0$, but $U(G)_{(j, l),(i, j)}=0$ since $i \neq l$.

The support of an $n \times n$ matrix $M$, denoted by $\underline{M}$, is the $n \times n(0,1)$-matrix with elements defined as follows:

$$
\underline{M}_{i, j}:= \begin{cases}1 & \text { if } M_{i, j} \neq 0 \\ 0 & \text { otherwise }\end{cases}
$$

Let $M(G)$ be the adjacency matrix of a graph (digraph) $G$. The line digraph of a digraph $D$, denoted by $\vec{L} D$, is the digraph defined as follows: $V(\vec{L} D)=A(D)$ and 
$((i, j),(k, l)) \in A(\vec{L} D)$ if and only if $j=k$. If $d(i)>2$ for every $i \in V(G)$ then one can verify that $\underline{U(G)}=M\left(\vec{L} D_{G}\right)[12]$.

Graphs (or digraphs) $G$ and $H$ are isomorphic (written $G \cong H$ ) if there is a permutation matrix $P$ such that $M(G)=P M(H) P^{-1}$, where $M(G)$ and $M(H)$ are the adjacency matrices of the graphs $G$ and $H$, respectively. Harary and Norman [7] proved that if $D$ and $F$ are digraphs without sources or sinks then $\vec{L} D \cong \vec{L} F$ if and only if $D \cong F$ $(i \in V(D)$ is a source if there is no $j$ such that $(j, i) \in A(D)$; a sink if there is no $j$ such that $(i, j) \notin A(D))$. Since $D_{G}$ is without sources or sinks, it follows that $G \cong H$ if and only if $U(G)=Q U(H) Q^{-1}$, for some permutation matrix $Q$. According to Definition 1, this fact is sufficient to ensure that $G \cong H$ if and only if $U(G)=Q U(H) Q^{-1}$, for some permutation matrix $Q$. In words, $U(G)$ "faithfully" represents $G$.

The spectrum of a matrix $M$ is the multiset of the eigenvalues of $M$, and is denoted by $\operatorname{Sp}(M)=\left\{\left[\lambda_{i}\right]^{m_{i}}: 1 \leq i \leq m\right\}$, where $\lambda_{1}, \lambda_{2}, \ldots, \lambda_{k}$ are the eigenvalues of $M$ and $m_{1}, m_{2}, \ldots, m_{k}$ their respective multiplicities. Let $T(G)$ be the matrix with $i j$-th entry

$$
T(G)_{i, j}:= \begin{cases}\frac{1}{d(j)}, & \text { if } M(G)_{i, j}=1 \\ 0, & \text { otherwise. }\end{cases}
$$

We can obtain $\operatorname{Sp}(U(G))$ from $\operatorname{Sp}(T(G))$, as shown in the next result.

Proposition 2.2 Let $G$ be a graph on $n$ vertices and $m$ edges. The matrix $U(G)$ has $2 n$ eigenvalues of the form

$$
\lambda=\lambda_{T} \pm i \sqrt{1-\lambda_{T}^{2}}
$$

where $\lambda_{T}$ is an eigenvalue of the matrix $T(G)$. The remaining $2(m-n)$ eigenvalues of $U(G)$ are \pm 1 with equal multiplicities.

Proof. Let $M=M(G), U=(G)$ and $T=T(G)$. Since $G$ is undirected, we have $M_{i, j}^{2}=M_{i, j} M_{j, i}=M_{i, j}$. By Definition 1,

$$
U_{(i, j),(k, l)}=M_{i, j} M_{k, l} \delta_{j, k}\left(\frac{2}{d(k)}-\delta_{i, l}\right) .
$$

Let $\mathbf{u}$ be an eigenvector of $U$ with eigenvalue $\lambda$ (notice that the entries of $\mathbf{u}$ have two indices as it is for the rows and the columns of $U$ ). By making use of this equation, we can write

$$
\begin{aligned}
\lambda u_{(i, j)} & =\sum_{k, l} U_{(i, j),(k, l)} u_{(k, l)} \\
& =2 \frac{M_{i, j}}{d(j)} \sum_{l} M_{j, l} u_{(j, l)}-M_{i, j} u_{(j, i)} .
\end{aligned}
$$


Let $\mathbf{t}$ be an eigenvector of $T$ with eigenvalue $\lambda_{T}$. Define

$$
u_{(i, j)}=\frac{M_{i, j} t_{j}}{d(j)}-\lambda^{*} \frac{M_{i, j} t_{i}}{d(i)} .
$$

Then

$$
\begin{aligned}
& 2 \frac{M_{i, j}}{d(j)} \sum_{l} M_{j, l} u_{(j, l)}-M_{i, j} u_{(j, i)} \\
& =\frac{M_{i, j} t_{j}}{d(j)}\left(2 \lambda_{T}-\lambda^{*}\right)-M_{i, j} \frac{t_{i}}{d(i)} .
\end{aligned}
$$

Since $U$ is real-orthogonal, its eigenvalues have magnitude 1 . It follows that

$$
\lambda u_{(i, j)}=\lambda\left(\frac{M_{i, j} t_{j}}{d_{j}}\left(2 \lambda^{*} \lambda_{T}-\left(\lambda^{*}\right)^{2}\right)-\lambda^{*} M_{i, j} \frac{t_{i}}{d_{i}}\right) .
$$

Therefore, $\mathbf{u}$ is an eigenvector of $U$ if $2 \lambda^{*} \lambda_{T}-\left(\lambda^{*}\right)^{2}=1$ or $\lambda+\lambda^{*}=2 \lambda_{T}\left(\lambda^{*}\right.$ is the complex conjugate of $\lambda$ ). It is easy to verify that the remaining eigenvalues are \pm 1 .

Two graphs $G$ and $H$ are said to be cospectral (or isospectral), with respect to a given matrix representation, if the spectra of the matrices representing the graphs are identical (see, e.g., [16]). For example, let $G$ and $H$ such that

$$
M(G)=\left(\begin{array}{ccccc}
0 & 1 & 1 & 0 & 0 \\
1 & 0 & 0 & 1 & 0 \\
1 & 0 & 0 & 1 & 0 \\
0 & 1 & 1 & 0 & 0 \\
0 & 0 & 0 & 0 & 0
\end{array}\right)
$$

and

$$
M(H)=\left(\begin{array}{lllll}
0 & 0 & 0 & 0 & 1 \\
0 & 0 & 0 & 0 & 1 \\
0 & 0 & 0 & 0 & 1 \\
0 & 0 & 0 & 0 & 1 \\
1 & 1 & 1 & 1 & 0
\end{array}\right) .
$$

Then $\operatorname{Sp}(M(G))=\operatorname{Sp}(M(H))$. The following corollary is a straightforward consequence of Proposition 2.2.

Corollary 2.3 Given graphs $G$ and $H, \operatorname{Sp}(U(G))=\operatorname{Sp}(U(H))$ iff $\operatorname{Sp}(T(G))=\operatorname{Sp}(T(H))$.

If $G \cong H$ then $\operatorname{Sp}(M(G))=\operatorname{Sp}(M(H))$, but the converse is not necessarily true. This is the case for the graphs $G$ and $H$ considered in the above example, since $\operatorname{Sp}(M(G))=$ $\operatorname{Sp}(M(H))=\left\{[-2]^{1},[0]^{3},[2]^{2}\right\}$ but, of course, $G \nsubseteq H$. Also, $\operatorname{Sp}(T(G))=\operatorname{Sp}(T(H))$ does not imply that $G \cong H$. For instance, if $G$ is a $k$-regular graph then $\operatorname{Sp}(T(G))=\left\{\left[\frac{1}{k} \lambda_{i}\right]^{m_{i}}\right.$ : $1 \leq i \leq k\}$, where $\operatorname{Sp}(M(G))=\left\{\left[\lambda_{i}\right]^{m_{i}}: 1 \leq i \leq k\right\}$. So, for two $k$-regular graphs $G$ 
and $H, \operatorname{Sp}(M(G))=\operatorname{Sp}(M(H))$ if and only if $\operatorname{Sp}(T(G))=\operatorname{Sp}(T(H))$, or, equivalently, $\operatorname{Sp}(U(G))=\operatorname{Sp}(U(H))$. Two non-isomorphic $k$-regular cospectral graphs $G$ and $H$ are then not distinguished by the spectra of $U(G)$ and $U(H)$.

Now, let $M$ and $N$ be matrices such that $\operatorname{Sp}(M)=\operatorname{Sp}(N)=\left\{\left[\lambda_{i}\right]^{m_{i}}: 1 \leq i \leq n\right\}$. For any natural number $p, \operatorname{Sp}\left(M^{p}\right)=\operatorname{Sp}\left(N^{p}\right)=\left\{\left[\lambda_{i}^{p}\right]^{m_{i}}: 1 \leq i \leq n\right\}$, but it is not necessarily the case that $\operatorname{Sp}\left(\underline{M^{p}}\right)=\operatorname{Sp}\left(\underline{N^{p}}\right)$. This is again the case of the graphs $G$ and $H$ in the above example:

$$
\begin{aligned}
& \underline{M(G)^{2}}=\left(\begin{array}{ccccc}
1 & 0 & 0 & 1 & 0 \\
0 & 1 & 1 & 0 & 0 \\
0 & 1 & 1 & 0 & 0 \\
1 & 0 & 0 & 1 & 0 \\
0 & 0 & 0 & 0 & 0
\end{array}\right) ; \\
& \underline{M(H)^{2}}=\left(\begin{array}{lllll}
1 & 1 & 1 & 1 & 0 \\
1 & 1 & 1 & 1 & 0 \\
1 & 1 & 1 & 1 & 0 \\
1 & 1 & 1 & 1 & 0 \\
0 & 0 & 0 & 0 & 1
\end{array}\right) .
\end{aligned}
$$

$\operatorname{Sp}\left(\underline{M(G)^{2}}\right)=\left\{[0]^{3},[2]^{2}\right\}$ and $\operatorname{Sp}\left(\underline{\left.M(H)^{2}\right)}=\left\{[0]^{3},[1],[4]\right\}\right.$. The entry $M(G)_{i, j}^{p}$ equals the number of paths of length $p$ between vertices $i$ and $j$. Note that $M(G)_{1,1}^{2}=2$ and $\underline{M(G)^{2}}{ }_{1,1}=1$. This implies that matrix $\underline{M(G)^{p}}$ contains generally less information about the structure of a graph $G$, since $\frac{M(G)^{p}}{i, j}=1$ if and only if the number of paths of length $p$ between vertices $i$ and $j$ is non-zero. Despite this fact, it is legitimate to ask the following question: given graphs $G$ and $H$ such that $\operatorname{Sp}(M(G))=\operatorname{Sp}(M(H))$ what conditions imply that $\operatorname{Sp}\left(M(G)^{p}\right) \neq \operatorname{Sp}\left(M(H)^{p}\right)$, for some $p$ ? If a graph $G$ with diameter $p$ is connected and non-bipartite then $\overline{M(G)^{p}}=J$, where $J$ denotes the all-ones matrix.

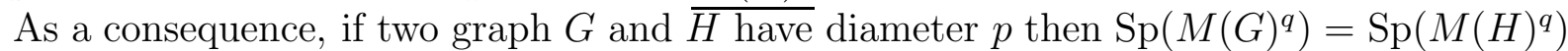
for every $q \geq p$, even if $\operatorname{Sp}(M(G)) \neq \operatorname{Sp}(M(H))$. The spectra $\operatorname{Sp}\left(\overline{\left.M(G)^{q}\right)}\right.$ and $\operatorname{Sp}\left(\overline{M(H)^{q}}\right)$ are indeed of no use in distinguishing $G$ and $H$ if these graphs have diameter 2.

Given a graph $G$, since the matrix $U(G)$ is real-orthogonal, the support of $U(G)^{p}$, for some $p$, is presumably different from the support of $M\left(\vec{L} D_{G}\right)^{p}$. This is because of the contribution of the negative entries in $U(G)$. On the basis of this observation, we define the following matrix.

Definition 2.4 Given a graph $G$, we denote by $S^{+}\left(U(G)^{p}\right)$ the matrix defined as follows:

$$
S^{+}\left(U(G)_{i, j}^{p}\right):= \begin{cases}1 & \text { if } U(G)_{i, j}^{p}>0 \\ 0 & \text { otherwise. }\end{cases}
$$

Suppose that $\operatorname{Sp}(M(G))=\operatorname{Sp}(M(H))$ and $G \nsucceq H$. What conditions need $G$ and $H$ to satisfy in order that $\operatorname{Sp}\left(S^{+}\left(U(G)^{p}\right)\right) \neq \operatorname{Sp}\left(S^{+}\left(U(H)^{p}\right)\right)$ for some $p \geq 2$ ? Strongly regular graphs seem to provide a good testing ground to approach this question. Strongly 
regular graphs have been investigated in many different contexts, including group theory, algebraic graph theory, design of experiments, finite geometries, error-correcting codes, etc. (see, e.g., $[2,3]$ ). The fastest known algorithm for testing the isomorphism of strongly regular graphs on $n$ vertices was designed by Spielman [15] and runs in $n^{O\left(n^{1 / 3} \log n\right)}$ number of steps. A strongly regular graph with parameters $(n, d, r, s)$ (for short, a $\operatorname{srg}(n, d, r, s)$ ) is a $d$-regular graph on $n$ vertices such that any two adjacent vertices have exactly $r$ common neighbours and any two nonadjacent vertices have exactly $s$ common neighbours [3]. Strongly regular graphs are interesting in our context principally for the following two reasons:

- For the strongly regular graphs $G$ and $H$ with identical sets of parameters, we have $\operatorname{Sp}(M(G))=\operatorname{Sp}(M(H))$ even if $G \nsubseteq H$. In particular, the adjacency matrix of a $\operatorname{srg}(n, d, r, s)$ has exactly three eigenvalues, $[d]^{1}>\left[e^{+}\right]^{m^{+}} \geq\left[e^{-}\right]^{m^{-}}$, such that $e^{+}=\frac{1}{2}(r-s+\sqrt{\Delta})$ and $e^{-}=\frac{1}{2}(r-s-\sqrt{\Delta})$, with $\left\{m^{+}, m^{-}\right\}=\frac{1}{2}\left(n-1 \pm \frac{2 k+(n-1)(s-r)}{\sqrt{\Delta}}\right)$ and $\Delta=(s-r)^{2}+4(d-s)$.

- The diameter of a connected strongly regular graph is 2 (see, e.g., [3]).

\section{On the spectra of $S^{+}(U(G)), S^{+}\left(U(G)^{2}\right)$ and $S^{+}\left(U(G)^{3}\right)$}

In this section, we give formulas to compute the eigenvalues of $S^{+}(U(G))$ and $S^{+}\left(U(G)^{2}\right)$ from the eigenvalues of $M(G)$, when $G$ is strongly regular. It follows that neither $S^{+}(U(G))$ nor $S^{+}\left(U(G)^{2}\right)$ is of any help in distinguishing $G$. The eigenvalues of $S^{+}\left(U(G)^{3}\right)$ do not depend entirely on the eigenvalues of $M(G)$ and it seems difficult to give a formula for $\operatorname{Sp}\left(S^{+}\left(U(G)^{3}\right)\right)$ in terms of properties of $G$. On the other hand we show how to construct $S^{+}\left(U(G)^{3}\right)$ from $G$. We conjecture that $\operatorname{Sp}\left(S^{+}\left(U(G)^{3}\right)\right)$ distinguishes $G$ from its cospectral mates.

Given a digraph $D$, let $P(D, x)=\operatorname{det}(x I-M(D))$ be the characteristic polynomial of $M(D)$. It is known that $[8,10]$

$$
P(\vec{L} D, x)=x^{|A(D)|-|V(D)|} P(D, x) .
$$

Then

$$
\operatorname{Sp}(\underline{U(G)})=\operatorname{Sp}(M(G)) \cup\left\{[0]^{n k-\left(1+m^{+}+m^{-}\right)}\right\} .
$$

The following two propositions show that the spectra of $S^{+}(U(G))$ and $S^{+}\left(U(G)^{2}\right)$ are determined by the parameters of $G$. Therefore the spectra of these matrices do not characterize $G$ itself.

Proposition 3.1 Let $G$ be a k-regular graph on $n$ vertices. Let $\mathbf{e}$ be an eigenvector of $M(G)$ with eigenvalue $\lambda_{M}$ and let $\mathbf{u}$ be an eigenvector of $S^{+}(U(G))$ with eigenvalue $\lambda$. Then $S^{+}(U(G))$ has $2 n$ eigenvalues of the form

$$
\lambda=\frac{\lambda_{M}}{2} \pm i \sqrt{k-1-\lambda_{M}^{2} / 4}
$$


with eigenvectors having entries

$$
u_{i, j}=M(G)_{i, j} \mathbf{e}_{j}-\frac{1}{\lambda} M(G)_{i, j} e_{i}
$$

The remaining $n(k-2)$ eigenvalues of $S^{+}(U(G))$ take the values \pm 1 .

Proof. Observe that

$$
S^{+}\left(U(G)_{(i, j),(k, l)}\right)=\left(1-\delta_{i, l}\right) M_{i, j} M_{k, l} \delta_{j, k} .
$$

We then have

$$
\begin{aligned}
& \sum_{(x, y)} S^{+}\left(U(G)_{(a, b),(x, y)}\right) u_{x, y} \\
& =M_{a, b} \sum_{y} M_{b, y} u_{b, y}-M_{a, b} u_{b, a} \\
& =M_{a, b} \sum_{y} M_{b, y} e_{y}-\frac{1}{\lambda} M_{a, b} e_{b} \\
& \sum_{y} M_{b, y}-M_{a, b} e_{a}+\frac{1}{\lambda} M_{a, b} e_{b} \\
& =M_{a, b} e_{b}(\lambda-(k-1) / \lambda)-M_{a, b} e_{a} \\
& =\lambda\left(M_{a, b} e_{b}-\frac{1}{\lambda} M_{a, b} e_{a}\right)
\end{aligned}
$$

Then these eigenvalues and eigenvectors account for $2 n$ of the eigenvalues and eigenvectors of $S^{+}(U(G))$. It is easy to verify that the remaining $n(k-2)$ eigenvalues take the values \pm 1 .

Proposition 3.2 Let $G$ be a k-regular graph on $n$ vertices. Let $\mathbf{e}$ be an eigenvector of $M(G)$ with eigenvalue $\lambda_{M}$ and let $\mathbf{u}$ be an eigenvector of $S^{+}\left(U(G)^{2}\right)$ with eigenvalue $\lambda$. Then $S^{+}\left(U(G)^{2}\right)$ has $2 n$ eigenvalues of the form

$$
\lambda=\frac{\lambda_{M}^{2}}{2}+2-k \pm i \lambda_{M} \sqrt{k-1-\lambda_{M}^{2} / 4}
$$

with eigenvectors having entries

$$
u_{i, j}=M(G)_{i, j} e_{j}-\frac{\lambda^{*}-2+k}{\lambda_{M}(k-1)} A_{i, j} e_{i}
$$

The remaining $n(k-2)$ eigenvalues of $S^{+}\left(U(G)^{2}\right)$ take the value 2 .

Proof. Observe that

$$
S^{+}\left(U(G)^{2}\right)=M_{i, j} M_{j, k} M_{k, l}\left(2 \delta_{i, k} \delta_{j, l}+1-\delta_{i, k}-\delta_{j, l}\right) .
$$


We then have

$$
\begin{aligned}
& \sum_{(x, y)} S^{+}\left(U(G)_{(a, b),(x, y)}\right) u_{x, y} \\
& =\sum_{x, y} M_{a, b} M_{b, x} M_{x, y}\left(2 \delta_{a, x} \delta_{b, y}+1-\delta_{a, x}-\delta_{b, y}\right) \\
& \left(M_{x, y} e_{y}-\frac{\lambda_{M}\left(\lambda^{*}-2+k\right)}{k-1} M_{x, y} e_{x}\right) \\
& =M_{a, b} e_{b}\left(\lambda_{M}^{2}-\lambda^{*}\right)-M_{a, b} e_{a} \\
& \left(\frac{(2-k)\left(\lambda^{*}-2+k\right)+\lambda_{M}^{2}(k-1)}{\lambda_{M}(k-1)}\right) \\
& =M_{a, b} e_{b} \lambda-M_{a, b} \mathbf{e}_{a} \\
& \left(\frac{\left(\lambda^{*}-2+k\right)(2-k+\lambda-2+k)}{\lambda_{M}(k-1)}\right) \\
& =\lambda u_{a, b} .
\end{aligned}
$$

We can also write

$$
\begin{aligned}
& \sum_{(x, y)} S^{+}\left(U(G)_{(a, b),(x, y)}\right) u_{(x, y)} \\
& =2 M_{a, b} u_{a, b}+M_{a, b} \sum_{x, y} M_{b, x} M_{x, y} u_{x, y} \\
& -M_{a, b} \sum_{y} M_{b, a} M_{a, y} u_{a, y}-M_{a, b} \sum_{x} M_{b, x} M_{x, b} u_{x, b}
\end{aligned}
$$

If

$$
\sum_{y} M_{a, y} u_{a, y}=0 \quad \text { and } \quad \sum_{y} M_{x, b} u_{x, b}=0
$$

then

$$
\sum_{(x, y)} S^{+}\left(U(G)_{(a, b),(x, y)}\right) u_{x, y}=2 M_{a, b} u_{a, b}
$$

and $\mathbf{u}$ is an eigenvector with eigenvalue 2 , provided that $M_{a, b}=0$ and then $u_{a, b}=0$.

The following observation allows us to construct $S^{+}\left(U(G)^{3}\right)$ directly from a strongly regular graph $G$, without the need of first constructing $U(G)$.

Proposition 3.3 Let $G$ be a $\operatorname{srg}(n, k, r, s)$. Then $S^{+}\left(U(G)_{(i, j),(l, m)}^{3}\right)=1$ if and only if one of the following conditions holds:

1. $i=m, j \neq l$ and

$$
s+(r-s) M(G)_{j, l}-k+\frac{k^{2}}{4}>0
$$

(which always holds if $i=m, j \neq l$ and $k>4$ ); 
2. $i=l, m \neq j$ and $M(G)_{j, m}<\frac{2 r}{k}$;

3. $i=l$ and $m=j$;

4. $i \neq l, m=j$ and $M(G)_{i, l}<\frac{2 r}{k}$;

5. $i \neq l, i \neq m, j \neq l, j \neq m$ and

$$
\frac{2}{k}\left[s+(r-s) M(G)_{j, l}\right]>M(G)_{i, l}+M(G)_{j, m} .
$$

Proof. Let $G$ be a $\operatorname{srg}(n, k, r, s)$. Let $A=M(G)$ and $U=U(G)$. We define the amplitude of a given path to be the product of the entries of $U$ corresponding to the transitions along the path. The entry $S^{+}\left(U(G)_{(i, j),(l, m)}^{3}\right)$ is given by the sum of the amplitudes of all possible paths of length three from $(i, j)$ to $(l, m)$. In what follows we count all these paths to give conditions for $S^{+}\left(U(G)_{(i, j),(l, m)}^{3}\right)=1$. We begin by observing that $i \neq j$ and $l \neq m$ since $G$ is a simple graph. The cases in the following table completely describe the possible ways in which the vertices $i, j, l$, and $m$ can be identified with one another:

\begin{tabular}{||l|l|l||}
\hline$i=m(\neq l)$ & $i=l(\neq m)$ & $i \neq m$ and $i \neq l$ \\
\hline Case A: $j=l$ & Case C: $j=m$ & Case E: $j=m(\neq l)$ \\
Case B: $j \neq l$ & Case D: $j \neq m$ & Case F: $j=l \neq m)$ \\
& & Case G: $j \neq m$ and $j \neq l$ \\
\hline
\end{tabular}

For each case we count the possible paths below and thus determine $U_{(i, j),(l, m)}^{3}$. Note that all paths of length three must be of the form

$$
(i, j) \rightarrow(j, x) \rightarrow(x, l) \rightarrow(l, m),
$$

where $x \in\{i, m\}$ or $x$ is some vertex other than $i, j, l$ or $m$.

Case $\mathbf{A}(i=m$ and $j \neq l)$ There is the unique path that takes place only on the vertices $\{i, j, l, m\}$ :

$$
(i, j) \rightarrow(j, i)=(j, m) \rightarrow(m, l) \rightarrow(l, m),
$$

and has amplitude $\frac{2}{k}\left(\frac{2}{k}-1\right)^{2}$. The other paths are of the form

$$
(i, j) \rightarrow(j, x) \rightarrow(x, l) \rightarrow(l, m), x \notin\{i, j, l, m\},
$$

which have amplitude $\frac{8}{k^{3}}$. If $\{j, l\} \in E(G)$ there are $r-1$ such paths, if $\{j, l\} \notin E(G)$ there are $s-1$. Thus,

$$
U_{(i, j),(l, m)}^{3}=\frac{2}{k}\left\{\left(\frac{2}{k}-1\right)^{2}+\frac{4}{k^{2}}\left[s+(r-s) A_{j, l}-1\right]\right\} .
$$

Case B $(i=m$ and $j=l)$ There is the unique path that takes place only on the vertices $\{i, j, l, m\}$ :

$$
(i, j) \rightarrow(j, i) \rightarrow(i, j) \rightarrow(j, i)=(l, m)
$$


which has amplitude $\left(\frac{2}{k}-1\right)^{3}$. There are $k$ other paths of the form

$$
(i, j) \rightarrow(j, x) \rightarrow(x, j) \rightarrow(j, i)=(l, m), x \notin\{i, j, l, m\}
$$

which have amplitude $\frac{8}{k^{3}}$. Thus,

$$
U_{(i, j),(l, m)}^{3}=\frac{2}{k}-1 .
$$

Case $\mathbf{C}(i=l$ and $j \neq m)$ There are no paths which take place only on the vertices $\{i, j, l, m\}$. There are paths

$$
(i, j) \rightarrow(j, x) \rightarrow(x, l) \rightarrow(l, m), x \notin\{i, j, l, m\}
$$

of amplitude $\frac{8}{k^{3}}$. If $\{j, m\} \in E(G)$ there are $r-1$ such paths, if $\{j, m\} \notin E(G)$ there are $r$. Thus,

$$
U_{(i, j),(l, m)}^{3}=\frac{4}{k^{2}}\left(\frac{2 r}{k}-A_{j, m}\right) .
$$

Case $\mathbf{D}(i=l$ and $j=m)$ There are no paths which take place only on the vertices $\{i, j, l, m\}$. There are $r$ paths

$$
(i, j) \rightarrow(j, x) \rightarrow(x, l) \rightarrow(l, m), x \notin\{i, j, l, m\}
$$

with amplitude $\frac{8}{k^{3}}$. Thus,

$$
U_{(i, j),(l, m)}^{3}=\frac{8}{k^{3}} r .
$$

Case $\mathbf{E}(i \neq m, i \neq l$ and $j=m)$ If $\{i, l\} \notin E(G)$ then there are no paths which take place only on the vertices $\{i, j, l, m\}$. However, there are $r$ paths

$$
(i, j) \rightarrow(j, x) \rightarrow(x, l) \rightarrow(l, m), x \notin\{i, j, l, m\}
$$

with amplitude $\frac{8}{k^{3}}$. If $\{i, l\} \in E(G)$ there is the unique path

$$
(i, j) \rightarrow(j, i) \rightarrow(i, l) \rightarrow(l, m)
$$

with amplitude $\frac{4}{k^{2}}\left(\frac{2}{k}-1\right)$. When $\{i, l\} \in E(G)$ there are also $r-1$ paths

$$
(i, j) \rightarrow(j, x) \rightarrow(x, l) \rightarrow(l, m), x \notin\{i, j, l, m\}
$$

with amplitude $\frac{8}{k^{3}}$. Thus,

$$
U_{(i, j),(l, m)}^{3}=\frac{4}{k^{2}}\left(\frac{2 r}{k}-A_{i, l}\right) .
$$

Case $\mathbf{F}(i \neq m, i \neq l$ and $j=l)$ There are two paths

$$
(i, j) \rightarrow(j, i) \rightarrow(i, j) \rightarrow(j, m)=(l, m)
$$


and

$$
(i, j) \rightarrow(j, l) \rightarrow(l, j) \rightarrow(j, m)=(l, m)
$$

each of amplitude $\frac{2}{k}\left(\frac{2}{k}-1\right)^{2}$. There are also $k-2$ paths

$$
(i, j) \rightarrow(j, x) \rightarrow(x, l) \rightarrow(l, m), x \notin\{i, j, l, m\}
$$

with amplitude $\frac{4}{k^{2}}\left(\frac{2}{k}-1\right)$. Thus,

$$
U_{(i, j),(l, m)}^{3}=0 \text {. }
$$

Case $\mathbf{G}(i \neq m, i \neq l, j \neq l$ and $j \neq l)$ We consider the cases $\{j, l\} \in E(G)$ and $\{j, l\} \notin E(G)$ separately. Firstly, we consider the case $\{j, l\} \in E(G)$. There are $r-A_{i, l}-A_{j, m}$ paths

$$
(i, j) \rightarrow(j, x) \rightarrow(x, l) \rightarrow(l, m), x \notin\{i, j, l, m\}
$$

of amplitude $\frac{8}{k^{3}}$. There are also $A_{i, l}+A_{j, m}$ paths of amplitude $\frac{4}{k^{2}}\left(\frac{2}{k}-1\right)$. If they exist, these are

$$
(i, j) \rightarrow(j, m) \rightarrow(m, l) \rightarrow(l, m)
$$

and

$$
(i, j) \rightarrow(j, i) \rightarrow(i, l) \rightarrow(l, m) .
$$

Now, if $\{j, l\} \notin E(G)$ the analysis is identical but with $s$ replacing $r$. Thus,

$$
U_{(i, j),(l, m)}^{3}=\frac{4}{k^{2}}\left\{\frac{2}{k}\left[s+(r-s) A_{j, l}\right]-\left(A_{i, l}+A_{j, m}\right)\right\} .
$$

Notice that the conditions in the statement of the proposition are mutually exclusive. Each case corresponds to $U_{(i, j),(l, m)}^{3}>0$ for one of the cases A-F above. The condition 1 corresponds to the case A, 2 to C, 3 to D, 4 to E and 5 to F. We have $U_{(i, j),(l, m)}^{3} \leq 0$ for all the other cases.

We conclude with the following conjecture.

Conjecture 3.4 Let $G$ and $H$ be strongly regular graphs with the same set of parameters. Then $G \cong H$ if and only if $S p\left(S^{+}\left(U(G)^{3}\right)\right)=S p\left(S^{+}\left(U(H)^{3}\right)\right)$.

If the conjecture is true then isomorphism of strongly regular graphs can be tested in polynomial time.

\section{Computations}

The table below contains the parameters of the strongly regular graphs for which we have tested the validity of the conjecture. We have tested also the complements of these graphs. In all cases the conjecture held. The number of non-isomorphic $\operatorname{srg}(n, k, r, s)$ is denoted by $N(n, k, r, s)$. The graphs $\operatorname{srg}(16,9,4,6)$ were obtained from [9]; all other graphs where obtained from [14]: 


\begin{tabular}{||l|l||l|l||}
\hline$(n, k, r, s)$ & $N(n, k, r, s)$ & $(n, k, r, s)$ & $N(n, k, r, s)$ \\
\hline \hline$(16,6,2,2)$ & 2 & $(35,18,9,9)$ & 227 \\
$(16,9,4,6)$ & 2 & $(36,14,4,6)$ & 180 \\
$(25,12,5,6)$ & 15 & $(36,15,6,6)$ & 32,548 \\
$(26,10,3,4)$ & 10 & $(40,12,2,4)$ & 28 \\
$(28,12,6,4)$ & 4 & $(45,12,3,3)$ & 78 \\
$(29,14,6,7)$ & 41 & $(64,18,2,6)$ & 167 \\
\hline
\end{tabular}

The method that we have described appears then to be successful for strongly regular graphs. In general, there are cases in which the method fails. We have verified that $\operatorname{Sp}\left(S^{+}\left(U(G)^{3}\right)\right)$ distinguishes all regular graphs up to 13 vertices. However, we have found 4-regular graphs on 14 vertices which are not distinguished by our method. Two graphs for which the method fails are drawn in the figure below:

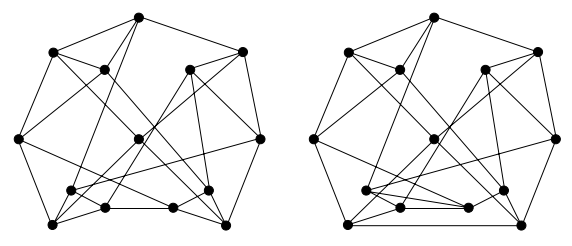

The computer programmes used for our calculations can be downloaded from www-users.cs.york.ac.uk/ ^wilson/qwalks.html

or requested from the authors. These are MATLAB and C codes.

Acknowledgments We would like to thank Lalit Jain, Chris Godsil, Toufik Mansour, Peter Cameron and Dennis Shasha for their interest in this work. We would like to thank the anonymous referees for their help in improving the content and the presentation of the paper. One of the referees noted that there are 3-regular graphs on 16 vertices that are not distinguished by the technique described in the paper.

\section{References}

[1] A. Ambainis, Quantum walks and their algorithmic applications, quant-ph/0403120.

[2] A. E. Brouwer, Strongly regular graphs, in: The CRC Handbook of Combinatorial Designs (eds.: Colbourn and Dinitz), 667-685, CRC Press, 1996.

[3] P. J. Cameron, Strongly regular graphs, www.maths.qmw.ac.uk/〜pjc/preprints.

[4] A. M. Duval, A directed graph version of strongly regular graphs, J. Combin. Theory Ser. A 47 (1988), no. 1, 71-100. 
[5] C. Godsil and G. Royle, Algebraic Graph Theory, Graduate Texts in Mathematics, 207, Springer-Verlag, New York, 2001.

[6] V. Gudkov and S. Nussinov, Graph equivalence and characterization via a continuous evolution of a physical analog, cond-mat/0209112.

[7] F. Harary, Frank and R. Z. Norman, Some properties of line digraphs, Rend. Circ. Mat. Palermo (2) 9 1960, 161-168.

[8] G. N. Lin, Guo Ning and F. J. Zhang, Characteristic polynomials of directed line graphs, and a class of directed graphs with the same spectrum (Chinese), Kexue Tongbao (Chinese) 28 (1983), no. 22, 1348-1350.

[9] B. McKay, cs .anu.edu.au/ bdm/data/latin.html.

[10] V. R. Rosenfeld, Some spectral properties of the arc-graph, Match No. 43 (2001), $41-48$.

[11] T. Rudolph, Constructing physically intuitive graph invariants, quant-ph/0206068.

[12] S. Severini, The underlying digraph of a coined quantum random walk, ERATO Conference in Quantum Information Science, Tokyo, Japan, 2003, quant-ph/0210055.

[13] S.-Y. Shiau, R. Robert, and S. N. Coppersmith, Physically-motivated dynamical algorithms for the graph isomorphism problem, quant-ph/0312170.

[14] T. Spence, www.maths.gla.ac.uk/ es/srgraphs.html.

[15] D. A. Spielman, Faster isomorphism testing of strongly regular graphs, Proceedings of the Twenty-eighth Annual ACM Symposium on the Theory of Computing (Philadelphia, PA, 1996), 576-584, ACM, New York, 1996.

[16] E. R. van Dam and W. H. Haemers, Which graphs are determined by their spectrum? Special issue on the Combinatorial Matrix Theory Conference (Pohang, 2002), Linear Algebra Appl. 373 (2003), 241-272. 